Ann. Zootech., I965, 14 (3), 285-297.

\title{
ESSAI D'APPLICATION D'UN TEST DE CONVERGENCE DE DROITES DE RÉGRESSION A LA CROISSANCE DES PORCELETS
}

\author{
C. LEGAULT, J.-C. CANONGE \\ Station centrale de Génétique animale, \\ Centre national de Recherches zootechniques, Jouy-en-Josas (Seine-et-Oise)
}

SOMMAIRE

Après vérification de la linéarité des courbes de croissance des porcelets pour des périodes brèves (deux à trois semaines) et compte tenu du fait que les animaux les plus lourds à un âge donné sont en général ceux qui ont la vitesse de croissance la plus élevée, nous avons fait l'hypothèse que pour une courte période, les courbes de croissance des animaux d'une population donnée peuvent être envisagées sous forme de faisceaux de droites concourantes.

La détermination des coordonnées du point de concours théorique qui se fait par la méthode des moindres carrés, ne prouve pas que les "droites de croissance " passent effectivement par ce point. Nous avons donc appliqué un test statistique de l'hypothèse de convergence de familles de droites de régression (RoBson et ATKINSON, r960) aux familles de "droites de croissance "

Les résultats, qui, dans leur ensemble, confirment la notion statistique de " foyer de croissance ", sont d'un grand intérêt pratique, pour la normalisation des résultats de pesées. Ils laissent donc entrevoir une possibilité d'exploitation simple des contrôles extensifs effectués dans les élevages, afin, par exemple, d'obtenir pour les porcelets le poids à $21 \mathrm{j}$ ou à $5^{6} \mathrm{j}$ à partir de pesées effectuées au voisinage de ces âges.

\section{I. - INTRODUCTION}

La croissance d'un animal domestique présente un certain nombre de phases caractéristiques de son développement biologique (naissance, sevrage, puberté) et de son exploitation zootechnique (changement de régime alimentaire, commercialisation). Le contrôle officiel à un âge fixe des performances individuelles de tous 
les sujets d'un élevage étant impossible dans la pratique, la détermination des poids de référence correspondants peut se faire de différentes manières :

- Sur la ligne de régression individuelle du poids sur l'âge lorsqu'on dispose d'au moins trois pesées par animal (fig. I. I.).

- Par interpolation linéaire lorsqu'on dispose de deux pesées encadrant l'âge de référence (fig. I. 2.); on fait alors l'hypothèse d'une croissance linéaire des animaux entre ces deux contrôles, celle-ci étant d'autant plus justifiée que l'intervalle de temps considéré est plus réduit.

- Sur la ligne de régression générale lorsqu'on ne dispose que d'un contrôle voisin de l'âge de référence. Dans ce cas, on fait 1'hypothèse que les croissances des animaux s'expriment par đes lignes de régression parallèles entre elles et parallèles à la ligne de régression générale (fig. I. 3.).

- Sur une droite qui passe par un point fixe en faisant l'hypothèse qu'à l'intérieur d'une période limitée, les courbes de croissance des animaux sont linéaires et concourent en un point $\mathrm{C}\left(\mathrm{X}_{\mathbf{0}}, \mathrm{Y}_{0}\right)$ (fig. $\mathrm{I}_{\text {. }}$.). Cette méthode, qui revient à exprimer le poids d'un animal à un âge donné par une fonction linéaire de sa vitesse de croissance au même âge, tient compte du fait que les animaux les plus lourds sont en général ceux qui ont la vitesse de croissance la plus élevée à l'âge considéré.
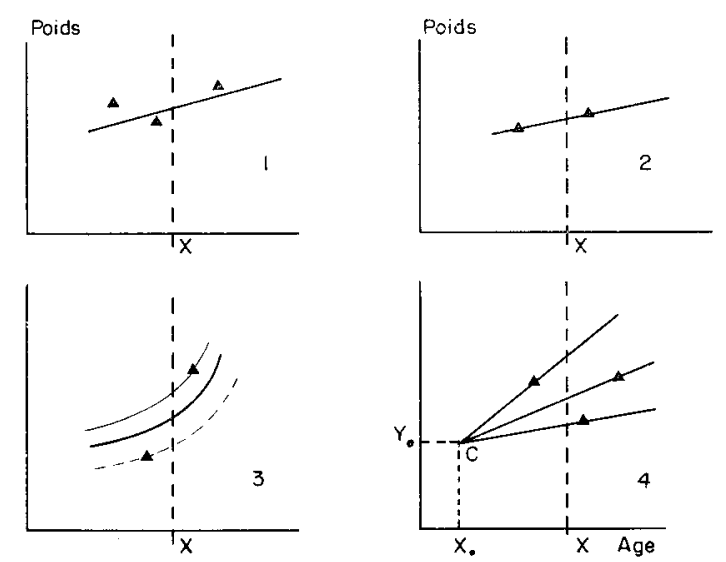

FıG. I. - Différents modes de normalisation du poids d'un animal dे un âge $\mathbf{x}$

TAYLOR et HAZEL (I955) ont fait une étude critique de différentes méthodes permettant la correction du poids des porcs à 1'âge de 54 jours à partir de pesées effectuées entre les $\mathrm{I} 34^{\mathrm{e}}$ et $\mathrm{I} 74^{\mathrm{e}}$ jours. Après avoir déterminé les coordonnées du point $\mathrm{C}$ par la méthode des moindres carrés, ces auteurs constatent que cette dernière méthode, en plus de sa simplicité, se révèle supérieure aux autres méthodes, les moyennes et les variances estimées étant respectivement les plus proches des moyennes et variances réelles. Signalons que MOLENAT et POULENC (I962) ont appliqué une méthode analogue pour estimer le poids à l'âge de 60 jours de porcelets pesés entre le $5^{\mathrm{e}}$ et $1 \mathrm{e} 8 \mathrm{o}^{\mathrm{e}}$ jour, après détermination graphique des coordonnées du point $C$.

Les auteurs que nous venons de citer ont apprécié la convergence des courbes de croissance d'une façon purement graphique. Les statisticiens, de leur côté, 
ont proposé des tests de 1'hypothèse de convergence de familles de droites de régression.

L'objet de cette étude est de présenter une justification statistique de cette notion de "foyer de croissance ", d'en montrer tout l'intérêt pour l'estimation de poids à âges fixes, et d'en fournir une illustration par une application à l'espèce porcine. Nous avons retenu à cet effet un test qui est dû à RoBson et ATKINSON (r960) et qui semble être actuellement le plus élaboré dans ses fondements statistiques; en contrepartie, il nécessite des calculs importants. Aussi, a-t-il fait l'objet d'un programme de calcul sur ordinateur I.B. M. I620 ( $\left.{ }^{(}\right)$.

\section{II. - BASES STATISTIQUES DU TEST DE CONVERGENCE D'UNE FAMILLE DE DROITES DE RÉGRESSION}

Tocher (1952), Williams (1953) et COCK (1963) ont proposé différents tests le l'hypothèse de convergence de droites de régression yui permettent également de déterminer les coordonnées $\left(X_{\theta}, Y_{n}\right)$ du point de concours $\mathcal{C}$. Ces trois tests consistent a minimiser la somme des carrés des écarts :

- soit des valeurs observées aux valeurs ajustées sur les droites supposées concourantes au point (' (Tocher et Williams) ;

soit du point $\mathrm{C}$ aux droites observées (Cock).

Le test que proposent Rorson et $\Lambda$ Thinson s'effectue en deux étapes qui répondent à deux questions :

I. Les droites sont-elles parallèles?

2. Si oui, sont-elles confondues? Sinon, sont-elles concourantes? (Dans les deux cas, ont-elles un point commun?) Rappelons brièvement le principe de ce test.

Soient :

- $\mathrm{D}_{i}$ l'estimation de la droite $\Delta_{i}, i$ variant de $\mathrm{r}$ à $t$.

- $t$ le nombre de droites.

- $n_{i}$ le nombre de couples $\left(x_{i}, y_{i j}\right)$ qui ont servi à estimer cette droite, avec $\sum_{i} n_{i}=n$.

- $b_{i}$ l'estimation de la pente $\beta_{i}$ de $\Delta_{i}$.

- $b_{w}$ la moyenne pondérée des $b_{i}$, chaque coefficient de pondération $w_{i}$ étant inversement proportionnel à la variance des $b_{i}$.

- $a_{i}$ l'estimation de l'ordonnée à l'origine $\alpha_{i}$ de la droite $\Delta_{i}$.

- $\hat{\alpha}_{i}$ l'estimation de $\alpha_{i}$ dans l'hypothèse d'homogénéité des $b_{i}$.

- $f_{m}$ un polynôme de degré $m, m$ variant de I à $t$ - I.

- $a_{q}$ et $q_{i}$ les termes correspondant respectivement à $b_{t}$ et $w_{i}$ dans le test de convergence.

- $s^{2}$ l'estimation de la variance résiduelle commune $\sigma^{2}$.

Le test de parallélisme a été emprunté à OstLe (1960). Dans l'hypothèse d'homogénéité de l'ensemble des $b_{i}$, la variable aléatoire :

$$
\mathrm{G}=\frac{\sum_{i} w_{i}\left(b_{i}-b_{\omega}\right)^{2}}{s^{2}(t-\mathrm{I})}
$$

est distribuée suivant une loi de $\mathbf{F}$ avec les degrés de liberté $\nu_{1}=t-\mathrm{I}$ et $\nu_{2}=n-2 t$.

L'originalité de la méthode de RoBSON et ATKINSON est liée à la décomposition du numérateur de $\mathrm{G}$ en $t$ - I termes, attachés chacun à un degré de liberté :

$$
\sum w_{i}\left(b_{i}-b_{w}\right)^{2}=\mathrm{B}_{1}^{2}+\cdots \mathrm{B}_{m^{2}}+\cdots \mathrm{B}_{t-1}{ }^{2}
$$

(1) Ce programme enregistré sous le nº 63033 est accompagné d'une notice d'utilisation déposée au Bureau de Calcul de la Station centrale de Génétique animale. 
Dans cette expression, le coefficient $\mathrm{B}_{m}$ provient d'une décomposition polynomiale orthogonale de $b_{i}$ telle que :

$$
b_{i}=b_{w}+\underset{m}{\stackrel{\Sigma}{*}} B_{m} f_{m}\left(\alpha_{i}\right)
$$

Lorsque les droites sont parallèles, l'expression $\frac{\mathrm{B}_{m^{2}}}{s^{2}}$ est distribuée suivant une loi de $\mathrm{F}$ avec les degrés de liberté $v_{1}=$ I et $v_{2}=n-2 t$. Le test de paraliélisme revient donc à effectuer $t-\mathrm{I}$ tests élémentaires, et les auteurs montrent que chacun d'eux est plus puissant que le test global.

Le test de convergence proprement dit consiste à vérifier que les droites ont un point commun situé sur l'axe des ordonnées; autrement dit, il revient à tester l'homogénéité de l'ensemble des $a_{i}$.

Le raisonnement précédent se répète en remplaçant $G_{r}$ par

$$
\mathrm{J}=\frac{\sum_{i} q_{i}\left(a_{i}-a_{q}\right)^{2}}{s^{2}(t-\mathrm{I})}
$$

et en écrivant : $\Sigma q_{i}\left(a_{i}-a_{q}\right)^{2}=A_{1}^{2}+\cdots A_{m}^{2}+\cdots A_{t-1}^{2}$

Nous proposons un seul apport dans l'application de ce test : il consiste, lorsque le point de convergence C des droites $\mathrm{D}$ ne se trouve pas sur l'axe des ordonnées, ì estimer l'abscisse $\left(\mathrm{X}_{0}\right)$ de ce point, s'il existe, par la méthode des moindres carrés.

$$
\mathrm{X}_{0}=\frac{t \sum_{i} a_{i} b_{i}-\sum_{i} a_{i} \sum_{i} b_{i}}{\left(\sum_{i} b_{i}\right)^{2}-t \sum_{i} \tilde{b}_{i}^{2}}
$$

On transporte ensuite l'origine des axes au point $\mathrm{C}_{\text {}}$ de l'axe OX tel que :

$$
\overline{\mathrm{OC}_{0}}=\mathrm{X}_{0}
$$

Nous n'avons pas étudié l'incidence de ce mode d'estimation de $\mathrm{X}_{0}$ sur la probabilité de rejeter l'hypothèse de convergence alors qu'elle est vraie, ou inversement.

\section{III. - MATÉRIEL ANIMAL ET CONSTITUTION DES CLASSES}

L'échantillon étudié représente cent portées de porcelets de race Large-White contrôlés à la Station de Recherches sur l't́levage des Porcs en 1959 et I960. Ces porcelets ont été pesés individuellement de la naissance à l'âge de deux mois, à des intervalles de temps variant de deux à cinq jours. La variable analysée est le poids moyen par portée entre les âges de six ct soixante jours, calculé à des intervalles de temps de trois jours par interpolation linéaire. Dans le calcul de ce poids moyen, les porcelets morts entre le sixième et le soixantième jour n'ont pas été considérés, de même que les portées d'effectif inférieur à six ou supérieur à onze porcelets.

Afin de satisfaire la condition de linéarité des régressions, il nous a paru nécessaire d'étudier dans un premier temps la forme de la courbe de croissance des porcelets jusqu'à l'âge de 60 jours.

A cet effet, nous avons établi l'é(quation de régression multiple dont les variables indépendantes sont les puissances croissantes de l'âge $(x)$ et la variable dépendante $(y)$ est le poids moyen des porcelets par portée. I.es fonctions du second, troisième ct quatrième degré sont respectivement :

$$
\begin{aligned}
& y=1,434+0,364 x+0,016 x^{2} \\
& y=1,084+0,604 x-0,014 x^{2}+0,001 x^{3} \\
& y=1,112+0,579 x+0,01026 x^{2}+0,00046 x^{3}+0,00001 x^{4} .
\end{aligned}
$$

La courbe de croissance observée ainsi que les courbes théoriques du second et du troisième degré sont représentées sur les figures 2. I. ct 2. 2. où $y$ cst exprimé en kilogrammes et $x$ en unités de temps de trois jours.

L'examen des résultats nous montre qu'une fonction du troisième degré exprime très bien 
la croissance moyenne des porcelets entre la naissance et le sevrage, le gain de précision obtenu en passant du troisième au quatrième degré étant négligeable.

En fonction de ces résultats, nous avons considéré indépendamment la croissance sur les trois périodes suivantes:

$\begin{array}{lr}\text { Période I } & 6-27 \text { jours } \\ \text { Période II } & 24-45 \text { jours } \\ \text { Période III } & 42-60 \text { jours }\end{array}$

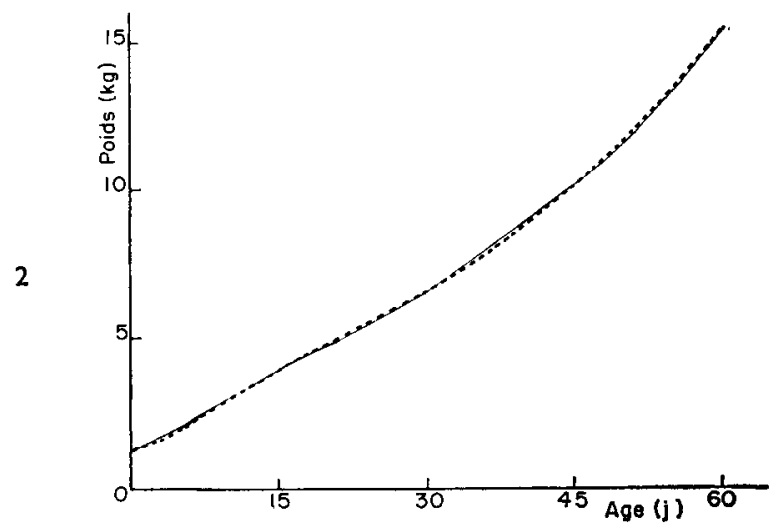

- Courbe de croissance théoriquac ( $3^{\mathrm{e}}$ degré)

_... - Courbe de croissance observée.

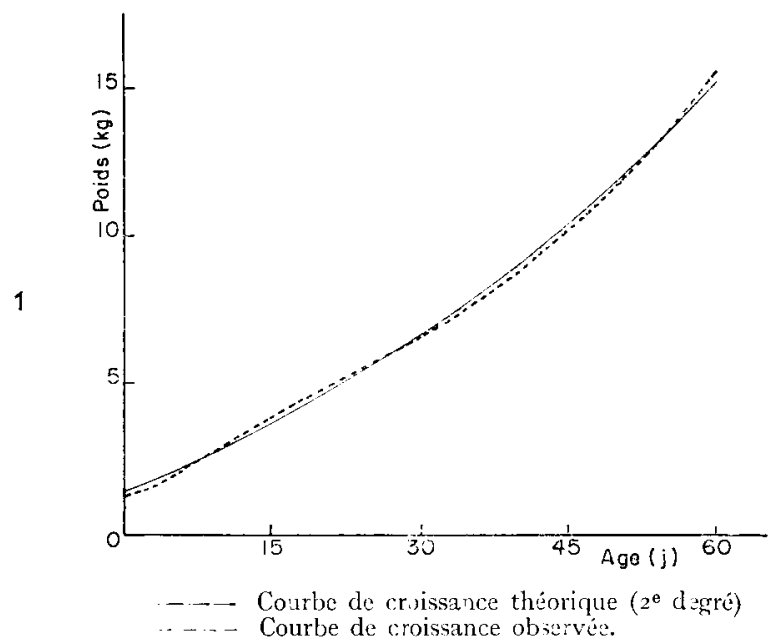

líg. 2. - - Courbe de croissance des porcelets de o à 60 jours

A l'intérieur de chacune de ces périodes, trois ensembles de droites $\mathrm{D}_{\boldsymbol{\xi}}$ ont été constitués à partir des mêmes données classées de trois manières différentes.

a) Les portées étant classées par ordre de poids moyen croissant aux âges respectifs de $2 \mathrm{I}$, 36 et 60 jours pour les périodes I, II et III, nous avons séparé le tiers moyen du tiers inférieur et du tiers supérieur de la distribution des fréquences, constituant ainsi trois classes dont les effectifs sont respectivement 33,34 et 33 portées.

b) Nous avons ensuite divisé l'étendue de la distribution en sept intervalles de classes égaux pour la période $\mathrm{I}$ et en dix pour les périodes II et III ; les effectifs $\mathrm{N}_{i}$ des classes ainsi déterminées sont reportés dans le tableau I.

c) La taille de la portée (constante durant la période de l'allaitement pour l'échantillon étudié) 
a servi enfin de critère de classification. Six classes correspondant aux portées de $6,7,8$, 9 , to et 1 ] porcelets ont des effectifs respectifs de I $2,15,28,27$, 10 et 8 .

Les courbes de croissance moyennes correspondant à ces trois modes de classification pour la fríriode I sont tracées sur les figures 3.1., 3.2., et 3.3.

1.

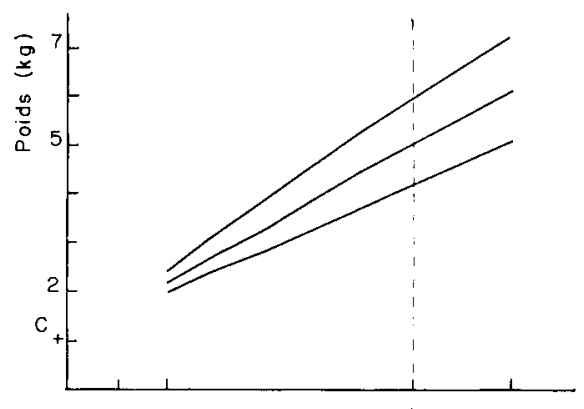

2.

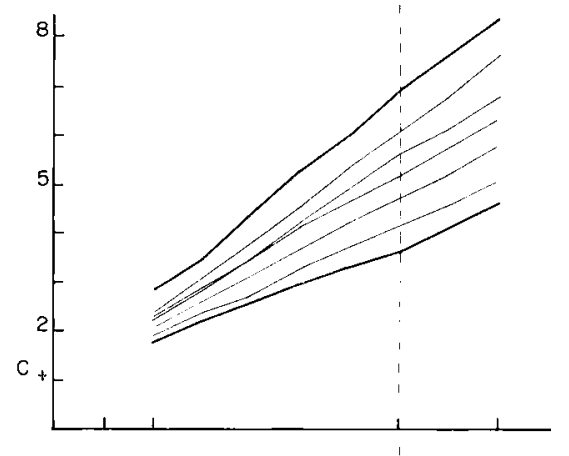

3.

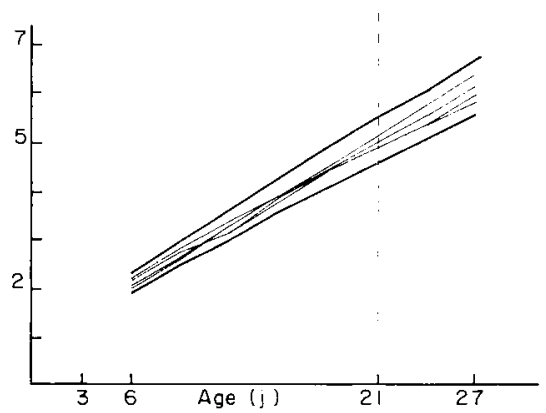

Fui. 3. - "Faisceaux de courbes de croissance" obtenus à partir d'un échantillon de 100 portées classées de trois manières différentes. (Période 6-27 jours)

\section{IV. - RÉSULTATS}

a) Vérification des conditions de validité du test de convergence de RoBson et AtKINSON

L'application de ce test suppose la normalité des distributions des poids à un âge donné, l'homogénéité des variances résiduelles, et la linéarité des régressions. La linéarité elle-même suppose, pour être testée, la normalité de la distribution de 
TABLEAU I

Résultats des tests de linéarité

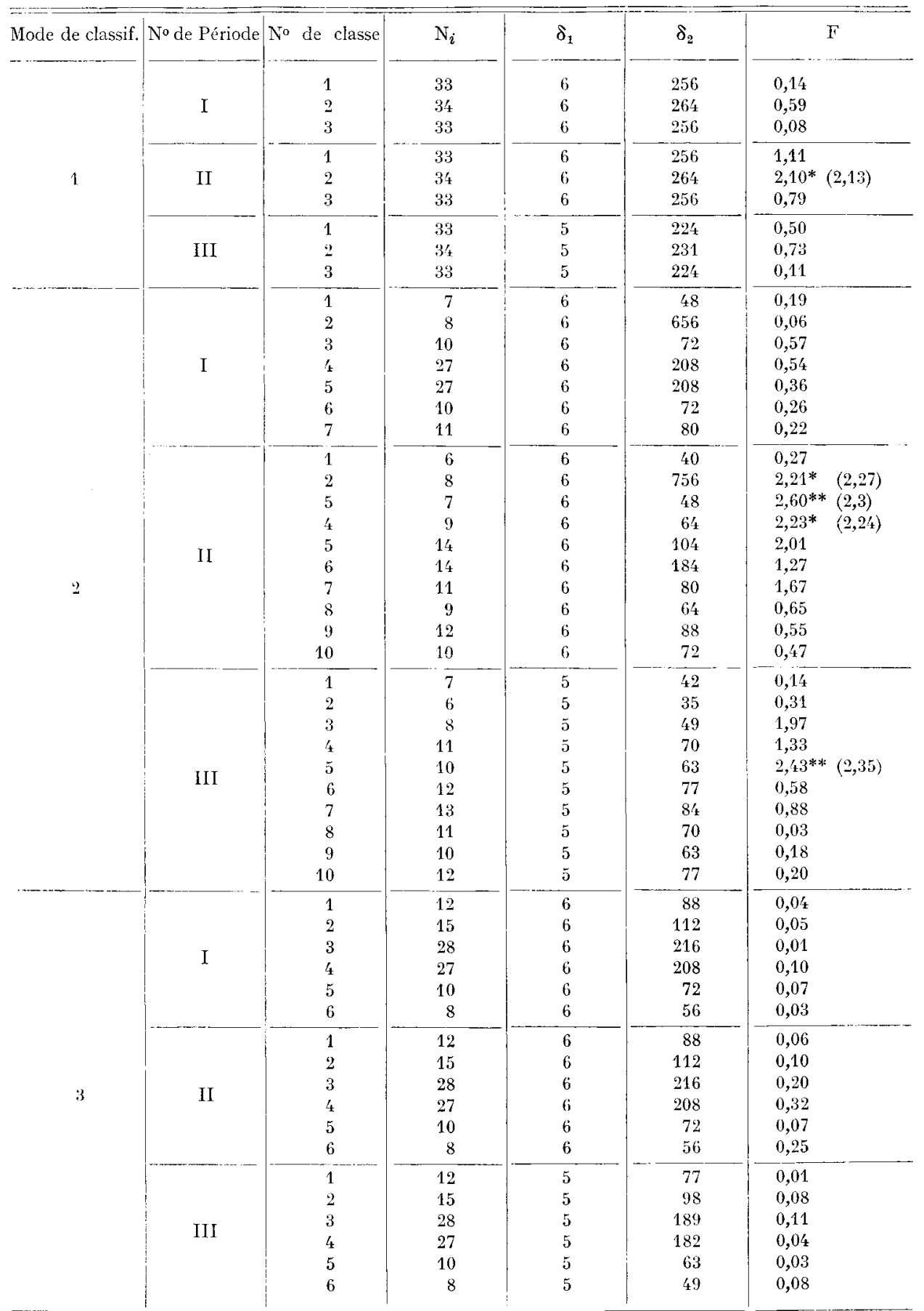

* Valeur de $\mathrm{F}$ voisine du seuil de signification à 5 p. 100.

** Valeur de F supérieure au seuil de signification à 5 p. 100. 
tous les poids d'une classe donnée. L'insuffisance des effectifs ne notrs a pas permis de le faire, et les résultats des tests de linéarité effectués selon la méthode décrite par Rouvier et CANONGE (I964) pour toutes les courbes étudiées sont rassemblés dans le tableau I où nous trouvons :

- $\mathrm{N}_{i}$ : nombre de portées par classe;

- $\delta_{2}$ et $\delta_{1}$ : nombres de degrés de liberté respectifs de la variance des moyennes de classe à partir de la droite de régression et de la variance résiduelle à partir des moyennes de classes.

- Les valeurs de $\mathrm{F}$ pour chacune des droites.

Les résultats nous montrent que, dans leur quasi-totalité, les éléments de courbe de croissance que nous avons analysés peuvent être assimilés à des droites. Nous n'observons en effet que deux cas de non-linéarité significatifs au seuil 0,05 sur les 54 cas correspondant à nos divers types de classification et cette proportion est environ celle à laquelle on pouvait s'attendre du simple fait du hasard.

\section{b) Résultats des tests}

Les résultats relatifs à la période I sont donnés en détail dans le tableau 2 alors que ceux concernant les périodes II et III sont résumés dans le tablearr 3 .

TABLEAU 2

Résultats relatifs à la période I (6-27 j)

\begin{tabular}{|c|c|c|c|c|c|c|c|c|c|c|c|}
\hline $\begin{array}{c}\text { Mode de } \\
\text { classification }\end{array}$ & $i$ & $\bar{y}_{i}$ & $b_{i}$ & $\begin{array}{l}\text { Nature } \\
\text { du }\end{array}$ & $v_{1}$ & $\nu_{2}$ & $F^{*}$ & IF & Conclusion & $x_{0}$ & $Y_{0}$ \\
\hline 1 & 2 & $\begin{array}{l}4,87 \\
4,15 \\
3,51\end{array}$ & $\begin{array}{l}0,69 \\
0,56 \\
0,45\end{array}$ & $\begin{array}{l}\text { CO } \\
\text { RP1 } \\
\text { RPR }\end{array}$ & 2 & $\begin{array}{l}794 \\
794 \\
794\end{array}$ & $\begin{array}{l}2,99 \\
3,8 x^{\prime} \\
3,8^{\prime}\end{array}$ & $\begin{array}{cc}\mathrm{P} & 99,99 \\
\mathrm{C} & 00,03 \\
\mathrm{P} & 99,99 \\
\mathrm{C} & 0,00 \\
\mathrm{P} & 0,007 \\
\mathrm{C} & 0,06\end{array}$ & C & $-0,42$ & 0,95 \\
\hline 2 & $\begin{array}{l}1 \\
2 \\
2 \\
3 \\
4 \\
5 \\
6 \\
7\end{array}$ & $\begin{array}{l}5,58 \\
\\
4,98 \\
4,53 \\
4,34 \\
3,96 \\
3,48 \\
3,13\end{array}$ & $\begin{array}{l}0,79 \\
\\
0,75 \\
0,67 \\
0,58 \\
8,53 \\
0,45 \\
0,39\end{array}$ & $\begin{array}{l}\mathrm{CO} \\
\mathrm{RP1} \\
\mathrm{RP2} \\
\mathrm{RPR}\end{array}$ & $\begin{array}{l}1 \\
1 \\
4\end{array}$ & $\begin{array}{r}786 \\
786 \\
786\end{array}$ & $\begin{array}{l}2,09 \\
\\
3,84 \\
3,84 \\
2,37\end{array}$ & $\begin{array}{rr}\text { P } & 99,99 \\
\text { C } & 3,74 \\
& \\
\text { P } & 99,99 \\
\text { C } & 0,38 \\
\text { P } & 0,13 \\
\text { C } & 0,04 \\
\text { P } & 6,36 \\
\text { C } & 5,50\end{array}$ & $\begin{array}{l}\text { Non } \mathrm{P} \\
\text { Non } \mathrm{C}\end{array}$ & $-0,60$ & 1,00 \\
\hline 3 & $\begin{array}{l}1 \\
2 \\
3 \\
4 \\
5 \\
6\end{array}$ & $\begin{array}{l}4,54 \\
4,25 \\
4,17 \\
4,13 \\
4,08 \\
3,79\end{array}$ & $\begin{array}{l}0,63 \\
0,62 \\
0,57 \\
0,52 \\
0,58 \\
0,52\end{array}$ & $\begin{array}{l}\text { CO } \\
\text { RP1 } \\
\text { RP2 } \\
\text { RPR }\end{array}$ & $\begin{array}{l}1 \\
3\end{array}$ & $\begin{array}{r}788 \\
788 \\
788 \\
788\end{array}$ & $\begin{array}{l}2,21 \\
3,84 \\
3,84 \\
2,60\end{array}$ & $\begin{array}{ll}\mathrm{P} & 2,73 \\
\mathrm{C} & 1,15 \\
\mathrm{P} & 7,39 \\
\mathrm{C} & 0,34 \\
\mathrm{P} & 0,17 \\
\mathrm{C} & 0,007 \\
\mathrm{P} & 2,04 \\
\mathrm{C} & 1,78\end{array}$ & $\mathrm{C}$ & 3,93 & 1,79 \\
\hline
\end{tabular}


Comme nous l'avons vu dans la partie théorique, le test de convergence s'effectue en deux étapes :

le test de non-parallélisme $(\mathrm{P})$;

le test de convergence proprement dit (C).

TABLEAU 3

Résultats relatifs aux périodes $I I$ et $I I I$

\begin{tabular}{|c|c|c|c|c|c|c|c|c|c|}
\hline Période & $\begin{array}{l}\text { Mode } \\
\text { de classi- } \\
\text { fication }\end{array}$ & $\begin{array}{l}\text { Hypothèse } \\
\text { testée }\end{array}$ & $v_{1}$ & $v_{2}$ & & $\begin{array}{l}\text { F } \\
\text { ximum }\end{array}$ & Conclusion & $\mathrm{X}_{0}$ & $Y_{0}$ \\
\hline \multirow{3}{*}{ II } & 1 & $\begin{array}{l}\mathrm{P} \\
\mathrm{C}\end{array}$ & $\begin{array}{l}1 \\
1\end{array}$ & $79 \prime$ & $\begin{array}{l}F_{R P 1} \\
F_{R P R}\end{array}$ & $\begin{array}{l}=99,99 \\
=\quad 0,25\end{array}$ & Non $\underset{\mathrm{C}}{\mathrm{P}}$ & 6,85 & 1,57 \\
\hline & 2 & $\begin{array}{l}\mathrm{P} \\
\mathrm{C}\end{array}$ & $\begin{array}{l}1 \\
7\end{array}$ & 780 & $\begin{array}{l}F_{R P r} \\
F_{R P R}\end{array}$ & $\begin{array}{l}=99,99 \\
=5,23\end{array}$ & $\begin{array}{l}\text { Non P } \\
\text { Non C }\end{array}$ & 8,19 & 1,85 \\
\hline & 3 & $\begin{array}{l}\mathrm{P} \\
\mathrm{C}\end{array}$ & $\begin{array}{l}3 \\
1\end{array}$ & 788 & $\begin{array}{l}F_{R P R} \\
F_{R P 1}\end{array}$ & $\begin{array}{l}=1,41 \\
=3,61\end{array}$ & $\begin{array}{r}\mathrm{P} \\
\mathrm{C} \\
\text { (confon- } \\
\text { dues) }\end{array}$ & 21,0 & 4,75 \\
\hline \multirow{3}{*}{ III } & 1 & $\begin{array}{l}\mathrm{P} \\
\mathrm{C}\end{array}$ & $\begin{array}{l}1 \\
1\end{array}$ & 694 & $\begin{array}{l}F_{R P 1} \\
F_{R / 1 R}\end{array}$ & $\begin{array}{l}=99,99 \\
=0,28\end{array}$ & Non $\begin{array}{r}\mathrm{P} \\
\mathrm{C}\end{array}$ & 26,55 & 4,12 \\
\hline & 2 & $\begin{array}{l}\mathrm{P} \\
\mathrm{C}\end{array}$ & $\begin{array}{l}1 \\
1\end{array}$ & 680 & $\begin{array}{l}\mathrm{F}_{\mathrm{RP} 1} \\
\mathrm{~F}_{\mathrm{RP} 2}\end{array}$ & $\begin{array}{l}=99,99 \\
=4,98\end{array}$ & $\begin{array}{l}\text { Non } P \\
\text { Non C }\end{array}$ & 26,5 & 3,77 \\
\hline & 3 & $\begin{array}{l}\mathrm{P} \\
\mathrm{C}\end{array}$ & $\begin{array}{l}1 \\
1\end{array}$ & 688 & $\begin{array}{l}F_{\text {RP1 }} \\
\mathrm{F}_{\mathrm{RP} 1}\end{array}$ & $\begin{array}{l}=4,38 \\
=0,92\end{array}$ & Non $\underset{\mathrm{C}}{\mathrm{P}}$ & 38,42 & 8,15 \\
\hline
\end{tabular}

Pour chacune de ces deux étapes nous avons fait figurer dans le tableau 2 les valeurs de $\mathrm{F}$ correspondant aux tests suivants :

- C.O. : test global d'homogénéité des $b_{i}(\mathrm{P})$ et des $a_{i}(\mathrm{C})$;

- RPI : test de nullité de $B_{1}(P)$ et de $A_{1}(C)$;

- RP2 : test de nullité de $B_{2}(P)$ et de $A_{2}(C)$;

- RPR : test global de nullité des coefficients restants $\left(B_{3}, \ldots B_{t-1}\right.$ et $\left.A_{3} \ldots A_{t-1}\right)$.

Dans ce tableau nous trouvons également:

- $\nu_{1}$ et $\nu_{2}$ : degrés de liberté correspondant à ces tests.

- $F^{*}$ : valeur de $F$ ayant la probabilité 0,05 d'être dépassée ;

- $\mathrm{X}_{0}$ : abscisse du point de convergence $\mathrm{C}$, exprimée en jours;

- $\mathrm{Y}_{\mathbf{0}}$ : ordonnée de $\mathrm{C}$ exprimée en $\mathrm{kg}$ et telle que :

$$
\mathrm{Y}_{0}=\frac{\sum_{i} b_{i} \sum_{i} a_{i} b_{i}-\sum_{i} a_{i} \sum_{i} b_{i}{ }^{2}}{\left(\sum_{i} b_{i}\right)^{2}-t \sum_{i} b_{i}{ }^{2}}
$$

$-b_{i}$ : pente de $D_{i}$ (égale aut gain pondéral par unité de temps de trois jours).

- $\bar{y}_{i}$ : poids moyen de la ième classe.

Annales de Zootechnie. - I965. 
En résumé, nous avons deux types de résultats selon le mode declassification considéré :

Io Modes de classification 1 et 3 .

Les tests établissent la convergence des droites de régression dans tous les cas. Ce résultat, prévisible au simple examen du graphique 2 . I., 1'est beaucoup moins à celui du graphique 2 . 3. Le troisième mode de classification aboutit en effet à un éventail de droites beaucoup plus resserré que le premier ; cet éventail est d'ailleurs complètement refermé pour la période II (droites confondues).

\section{$2^{0}$ Mode de classification 2.}

Les tests n'établissent la convergence des droites de régression en aucun cas. ce qui est difficilement décelable à l'examen du graphique 2. 2 .

\section{V. - DISCUSSION}

Il nous appartient, dans le cadre d'une discussion de ces premiers résultats, de rechercher les causes de leur diversité, et d'en déduire les meilleures conditions d'application du test.

\section{Analyse critique de l'essai}

Nous devons tout d'abord attirer l'attention sur le non-respect des conditions de normalité et d'homogénéité. L'étude d'une population de 5000 porcelets ayant révélé la normalité de la distribution du poids à 1'âge de six jours, il en est théoriquement de même de celle du poids moyen d'un échantillon de cent portées. Par contre, le choix de classes de faible effectif basé sur l'étendue de la distribution des fréquences nous éloigne des conditions précitées ( $2^{\mathrm{e}}$ mode de classification où une classe $n^{\prime}$ est représentée par exemple que par un effectif de 7 portées).

Un effectif total de cent portées s'est révélé trop faible pour constituer un essai valable de la méthode, la variation aléatoire des poids à l'intérieur des classes restant trop importante.

Enfin, signalons l'importance du mode de classification retenu ; mais en fonction de quel critère doit-on précisément le choisir?

\section{Recherche de la meilleure forme d'utilisation du test}

Une étude comparative de la valeur des critères de classification proprement dits, aurait nécessité, pour chacun d'eux, la répartition des données en un même nombre de classes. Cependant, l'objectif de notre étude étant 1'analyse du phénomène de " convergence " et de ses possibilités d'application, nous avons préféré faire varier le nombre de classes en nous efforçant d'adopter un type de classification facilement applicable dans la pratique.

Sur un plan purement graphique, la convergence sera d'autant plus facile 
à déceler que l'éventail des droites sera plus ouvert et que le nombre de droites sera plus réduit (I mor mode de classification).

Par contre, un critère biologique tel que taille de la portée, numéro de portée, type de croisement, etc. sera mieux adapté au respect des hypothèses de base, mais il entraînera en général un resserrement de l'éventail des droites $\left(3^{\mathrm{e}}\right.$ mode de classification).

En résumé, nous pouvons conseiller le respect des principes suivants :

\section{a) Choix du poids individuel des animaux.}

Le choix de la variable - le poids moyen des porcelets par portée -- diminue la variance des poids individuels et atténue le phénomène de la convergence des courbes de croissance. Nous devons remarquer à ce sujet que les pesées effectuées sous le contrôle du Herd-Book Large-White concernent la portée dans son ensemble et non les porcelets pris individuellement.

\section{b) Augmenter la taille de l'échantillon.}

La capacité du programme 63033 étant de ro ooo données (ro ooo pesées) nous pouvons accroître l'effectif animal en diminuant la longueur des périodes étudiées. $\mathrm{Si}$, par exemple, on ne retient que les cinq pesées qui encadrent 1'âge de référence, on pourra étudier simultanément 2 ooo animaux.

\section{c) Choisir des critères de classification biologiques.}

Ces critères pourront varier selon les espèces ; on peut proposer par exemple : la taille de la portée, le numéro de portée, le sexe pour les porcelets, le mode de naissance et le type d'alimentation pour les agneaux, le type de croisement pour les veaux de boucherie etc.

\section{d) Etendre l'analyse des coefficients de régression à d'autres phénomènes que la crois- sance.}

Toute relation linéaire entre deux grandeurs biologiques peut en effet s'étudier de la même façon que l'évolution du poids dans le temps (épaisseur du lard dorsal et le poids total du gras dans la carcasse par exemple).

e) Notons qu'une transformation logarithmique de la variable pourrait résoudre le problème de l'hétérogénéité des variances dans le cas où les coefficients de variation sont homogènes.

\section{Possibilités d'utilisation des résultats}

Les coordonnées $\left(\mathrm{X}_{\mathbf{9}}, \mathrm{Y}_{0}\right)$ du point $\mathrm{C}$ ne donnent qu'un ordre de grandeur des coordonnées réelles du point de convergence, et celles-ci petıvent d'ailleurs varier avec la race, le mode d'élevage, le troupeau, la saison, etc. Lorsque la convergence n'est pas établie statistiquement, C n'est que le point le "plus rapproché " de l'ensemble des droites. Un mode d'utilisation simple des résultats est illustré parl'exemple suivant : au cours de la période $I$ le point $\mathrm{C}$ se situe approximativement sur l'axe des ordonnées $\left(\mathrm{X}_{0}=0\right)$ à une distance de l'origine voisine de l'unité $\left(\mathrm{Y}_{0}=\mathrm{I}\right)$. Dans 
ces conditions, si un porcelet a un poids $y$ à un âge $x$ voisin de 2 I jours, le poids $\mathrm{Y}$ à 1'âge de $2 \mathrm{I}$ jours est donné par la relation :

$$
\mathrm{Y}=\mathrm{I}+\frac{2 \mathrm{r}(y-\mathrm{I})}{x}
$$

Si un porcelet pesait $5,85 \mathrm{~kg}$ à l'âge de 23 jours, à l'âge de $2 \mathrm{I}$ jours, il pesait :

$$
\mathrm{Y}=\mathrm{I}+\frac{2 \mathrm{I}(5,85-\mathrm{I})}{23}=5,43 \mathrm{~kg}
$$

Cette méthode laisse donc entrevoir une possibilité d'exploitation simple des contrôles extensifs non périodiques effectués sur des animaux d'élevage ou de boucherie à des stades bien définis de leur vie en vue d'obtenir :

Pour les bovins :

le poids à un âge fixe voisin du sevrage ou de la vente.

Pour les ovins :

poids à 30 jours ou 40 jours,

poids à roo jours (agneaux de boucherie).

Pour les porcins:

poids à 2I oul 60 jours,

épaisseur du lard dorsal an poids de Ioo $\mathrm{kg}$.

\section{VI. - CONCLUSION}

I a méthode statistique que nous avons étudiée permet d'envisager, pour des périodes relativement brèves, la croissance des animaux sous forme de droites à l'échelon individuel, et sous forme de faisceaux de droites convergentes à l'échelon d'une population. Ainsi, cette notion de "foyer de croissance " qui se dégage tout au long du développement de l'animal, reste à l'image du développement considéré dans son ensemble, les courbes de croissance se déroulant toutes sans discontinuité à partir de la cellule initiale que constitue l'œuf fécondé.

Bien qu'ils restent incomplets, les résultats de notre étude nous encouragent à poursuivte nos recherches en apportant les améliorations proposées dans la discussion.

Sur le plan pratique, l'utilisation de cette propriété a beaucoup d'intérêt tant par sa simplicité que par son vaste champ d'application. Flle permet en effet la transformation de données zootechniques brutes en données plus élaborées, susceptibles par conséquent d'être interprétées avec davantage de profit, que ce soit à des fins de gestion des troupeaux, ou à des fins génétiques. 


\title{
REMERCIEMENTS
}

Nous tenons à remercier nos collègues de la Station de Recherches sur l'Élevage des Porcs qui nous ont fourni les données analysées dans cette étude.

\section{SUMMARY}

\author{
APPLICATION OF A TEST OF CONVERGENCE OF REGRESSION LINES TO GROWTH IN PIGLETS
}

The well known fact that animals heavier at a given age are in general those with fastest rate of growth at that age suggested the hypothesis that within a restricted period their "growth curves ", the linearity of which had previously been verified, would meet at a point C.

The estimation of the coordinates of $\mathrm{C}$, by the method of least $\mathrm{s}$ (uares, did not prove that the growth curve did effectively pass through this point. Therefore a statistical test of the hypothesis, from the convergence of families of regression lines (ROBSON and ATKINSON, 1960), was applied to families of growth curves. The constitution of these groups of curves was made according to three criteria of classification, for three successive phases of growth.

Certain basic conditions could not be verified because of the small size of the samples, and the method of classification could cause some diversity in the results of the test. IIowever, taken together the results confirmed the idea of a "focus of growth " and the growth of a group of animals could be considered, for short periods ( 2 weeks) in the form of grouping of lines.

These results are of great practical interest for they show the possibility of the use of simple extensive records made on the farm for finding the weight of piglets at 21 or at 60 days from weights taken near those ages.

\section{RÉFÉRENCES BIBLIOGRAPHIQUES}

BYwatiks J. II., Wilitay D. S., 1935. A method of comparing rrowthiness in pigs weighed at different ages and subject to different treatments. Proc. amer. Soc. animal Prod., i 6.

Cock A. G., 1963. Genetical studies on growth and form in the fowl. Ginel. Res. Camb., 4, 167-192.

Molenat M., Poumenc J., 1962. Performances d'ülevage et sélection du porc Iarge-While. Bull. Tech. Inform. Ingrs. Serv. agric. Fr., I 7 I, 6г $7-684$.

Ostle 13., Ig60. Statistics in research. The Iowa Sta. Univ. Press, Ames, Iowa, 1. 136.

Robson D.S., AtKinson G. F., 1960. Individual degrees of freelom for 1 usting homogrencity of regression coefficients in a one-way analysis of covariance. Biomelrics, 16, 593-605.

Rouvier R., Canosge J. C., 1964. Réalisation et utilisation de programmes sur ordinateur qui permettent les calculs statistiques de base sur les données bionétriques. Ann. Zootech., 13, 379-387.

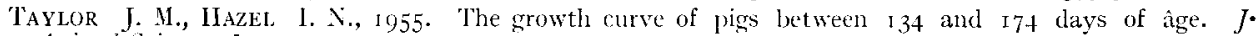
Animal Sci., 14, 4, 1 1 33 -1 139.

'Tociner K., I952. On the concurrence of a set of regression lines. Biomeirika, 39, rog-1 i 7 .

Whateex J. A., 1937. A method for comparing the growlh rates of pigs acrighed at different ages. Unpublished M. S. thesis, Ames, lowa (Iowa State Collerge), Cité par Taylor et IIAzel.

Williams E. S., 195.3. Tests of significance for concurrent regression lines. Biometrika, 40, 297-305. 